\title{
Simultaneous Transfemoral Transcatheter Aortic Valve Replacement of a Native Aortic Valve and Transapical Transcatheter Mitral Valve-in-Valve Replacement
}

\section{Abstract}

With Transcatheter Aortic Valve Replacement (TAVR) revolutionizing the treatment of high-surgical risk patients with severe aortic stenosis, Transcatheter Mitral Valve Replacement (TMVR) is slowly emerging as an off-label therapy for severe mitral valve disease. The case of a 70-year-old female with recurrent history of congestive heart failure has been described. This case demonstrates the simultaneous TAVR and off-label VIV TMVR is a reasonable alternative for highrisk surgical patients with stenotic native and failing bioprosthetic valves.

Keywords: Valvular heart disease; Transcatheter aortic valve replacement; Transcatheter mitral valve replacement

Received: June 26, 2017; Accepted: July 17, 2017; Published: July 24, 2017

\section{Introduction}

With Transcatheter Aortic Valve Replacement (TAVR) revolutionizing the treatment of high-surgical risk patients with severe aortic stenosis, Transcatheter Mitral Valve Replacement (TMVR) is slowly emerging as an off-label therapy for severe mitral valve disease [1-3]. Simultaneous TAVR and TMVR is less commonly performed. We describe the case of a 70-year-old female with New York Heart Association (NYHA) Class IV heart failure caused by severe native aortic stenosis and degenerated mitral prosthesis, who underwent successful simultaneous TAVR and valve-in-valve (VIV) TMVR.

\section{Case Presentation}

This is a 70-year-old female who presented with recurrent admissions for congestive heart failure. Her past medical history was significant for mitral valve replacement (\#29 mm Hancock II) for mitral stenosis in 2004, ASD closure, LAA ligation, MAZE procedure, sick sinus syndrome status-post pacemaker, paroxysmal atrial fibrillation, and multiple strokes. Over the past several months, she had progressive dyspnea and worsening peripheral edema, severely debilitating her activities of daily living. Physical examination was consistent with marked volume overload and cardiac exam consistent with mitral stenosis and aortic stenosis. One year prior, echocardiography showed moderate aortic stenosis with regurgitation and otherwise normal bioprosthetic mitral valve.

\author{
Tanya Doctorian ${ }^{1 *}$, \\ Daniel Serna², \\ Blanding Jones ${ }^{3}$, \\ Arti Choure ${ }^{1}$, Ramdlas Paii ${ }^{4}$ \\ Eric Chou ${ }^{1}$ and \\ William Mosley II ${ }^{1}$
}

1 Department of Cardiology, Kaiser Permanente Fontana Medical Centre, Fontana, CA, USA

2 Department of Cardiothoracic Surgery, Kaiser Permanente Fontana Medical Centre, Fontana, CA, USA

3 Department of Cardiothoracic Surgery, Kaiser Permanente Los Angeles Medical Centre. Los Angeles, CA, USA

4 Department of Cardiology, Dignity Health-St. Bernardine Medical Centre, San Bernardino, CA, USA

\section{*Corresponding author: Tanya Doctorian}

Đ tanya.doctorian@kp.org

Department of Cardiology, Kaiser Permanente Fontana Medical Centre, Fontana, CA, USA.

Tel: 909-302-5000

Citation: Doctorian T, Serna D, Jones B, Choure A, Pai R (2017) Simultaneous Transfemoral Transcatheter Aortic Valve Replacement of a Native Aortic Valve and Transapical Transcatheter Mitral Valve-inValve Replacement. Interv Cardiol J. Vol.3 No.2:19

The patient was admitted for diuresis and interrogation into possible worsening valvular disease contributing to her frequent heart failure exacerbations. Echocardiography revealed severe prosthetic mitral valve stenosis (mean gradient $22 \mathrm{mmHg}$ ), severe native aortic valve stenosis (mean gradient $38 \mathrm{mmHg}$, mean velocity $4.2 \mathrm{~m} / \mathrm{s}$, and aortic valve area $0.6 \mathrm{~cm}^{2}$ ), preserved left ventricular systolic function, dilated right atrium and right ventricle, and moderate right ventricular systolic dysfunction. Cardiac catheterization revealed severe pulmonary hypertension with pulmonary artery systolic pressure $(>90 \mathrm{mmHg}$ ) approaching 
systemic pressures. There was no epicardial coronary artery disease. CT showed aortic and prosthetic mitral valve calcification (Figure 1).

This patient was found to have NYHA Class IV heart failure caused by severe native aortic stenosis and severe prosthetic mitral stenosis. She was referred to cardiothoracic surgery for consideration of surgical replacement of both valves. She was deemed too high risk for traditional surgical replacement of both valves. Thus, a multidisciplinary decision was made to perform TAVR first followed by TMVR in the same setting under echocardiographic fluoroscopic guidance.

Left thoracotomy was performed prior to TAVR as well as heparinization to reduce the bleeding and to minimize transition time to the transapical approach. Heparinization followed left thoracotomy and insertion of the 14 F TAVR sheath. Successful TAVR via right femoral approach was first performed with a balloon expandable valve ( $23 \mathrm{~mm}$ Sapien 3, Edwards Lifesciences, Irvine, CA), followed by successful transapical VIV TMVR with a balloon expandable valve ( $26 \mathrm{~mm}$ Sapien XT, Edwards Lifesciences, Irvine, CA) placed in the inverted position (Figures 2-4). Postdeployment, the transvalvular pressure gradients normalized and pulmonary artery pressures improved significantly. The patient was discharged 7 days post-procedure with significant clinical improvement. At six-month and nine-month follow up appointments, the patient remains free of symptoms.

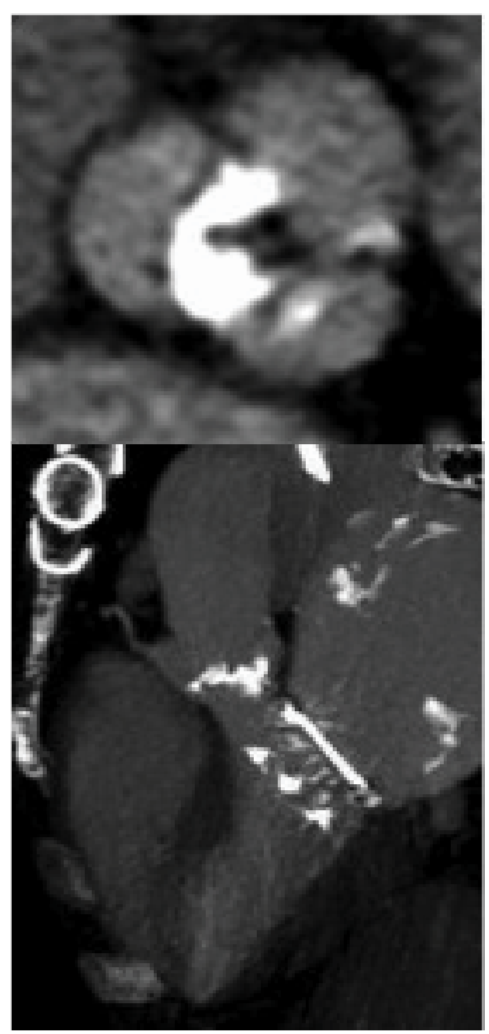

Figure 1 CT imaging: (A) Aortic valve stenosis with calcification; (B) Aortic and prosthetic mitral valve calcification.

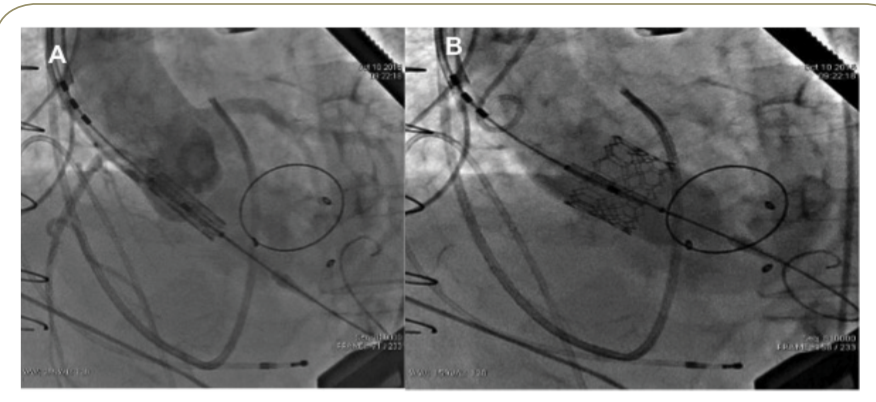

Figure 2 A balloon expandable $23 \mathrm{~mm}$ Edwards Sapien 3 valve was deployed in the aortic position via right transfemoral approach. Figure: (A) Pre-TAVR deployment; (B) PostTAVR deployment.

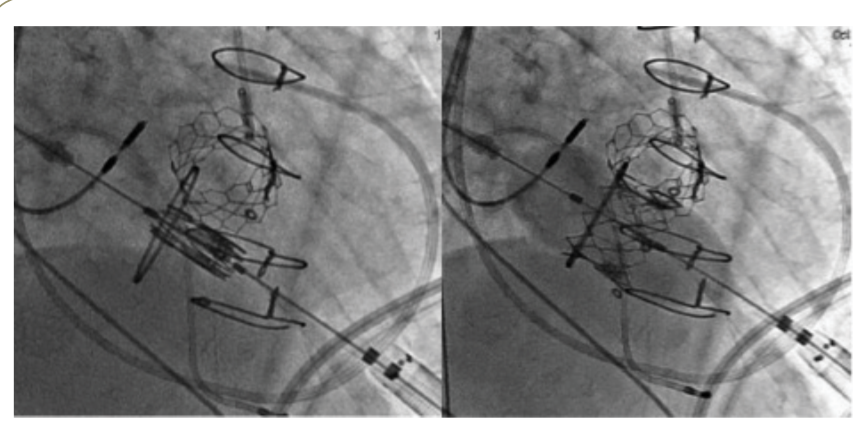

Figure 3 A balloon expandable $26 \mathrm{~mm}$ Edwards Sapien XT valve was then deployed (inverted position) in the mitral position via transapical approach. The valve was placed overlapping the previously placed bioprosthetic valve with $10 \%$ atrial extension for sufficient anchoring. (A) Pre-TMVR deployment; (B) Post-TMVR deployment; (C) Post-TAVR and VIV TMVR.

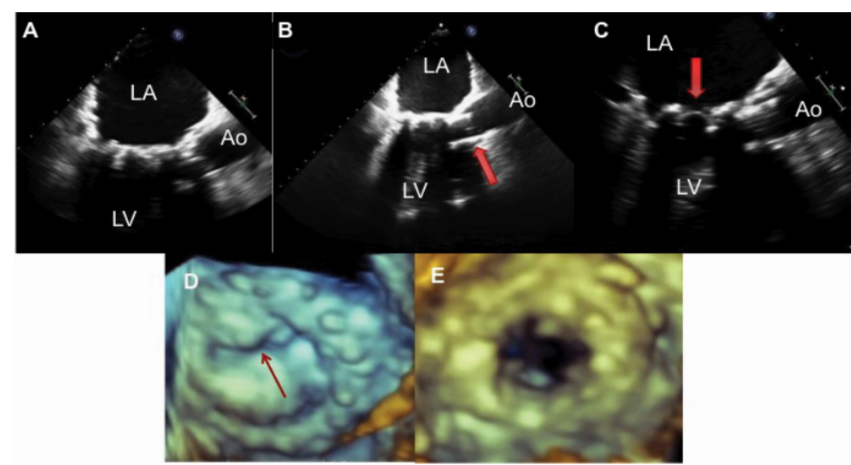

Figure 4 Intraoperative echocardiography showing: (A) Pre-TAVR, Pre-TMVR; (B) Post-TAVR (arrow), Pre-TMVR; (C) PostTAVR, Post-TMVR (arrow); (D) Severe bioprosthetic mitral stenosis (arrow); (E) Mitral valve after Edwards Sapien valve-in-valve replacement.

\section{Discussion}

In regard to our patient, several periprocedural considerations were made by the heart team. She was deemed too high risk for traditional surgical replacement of both valves for several reasons including severe right ventricular dysfunction, severely dilated 
right atrium and ventricle adherent to the sternum, and history of multiple strokes. Rather than forego intervention altogether or perform replacement of one valve, a multidisciplinary decision was made to perform a simultaneous transfemoral TAVR and a transapical VIV TMVR. With regards to the VIV TMVR, the transseptal approach, although less invasive, was not taken because the patient had a prior ASD closure and MAZE procedure with multiple sutures in the septum. Thus, we performed a transapical VIV TMVR. Furthermore, the aortic valve was replaced first, followed by mitral valve replacement. The converse order of valve replacement may have caused increased left ventricular pressures leading to left ventricular failure, potential left ventricular outflow tract obstruction, and mechanical damage to the mitral valve apparatus. Ten months post-procedure, our patient remains free of symptoms and has a significantly improved quality of life with no cardiac or all-cause hospital admissions.

Too often, patients have been denied treatment for valvular heart disease in the past due to being deemed too sick or too high surgical risk [1,2]. Advancements in transcatheter valvular treatment allow us to cost-effectively treat very sick patients with valvular heart disease and improve their quality of life [3]. TAVR is a widely recognized alternative treatment of severe aortic stenosis in intermediate to severe high surgical risk patients. While surgical mitral valve repair or replacement is still the preferred treatment for severe mitral regurgitation, several

\section{References}

1 Nishimura RA, Catherine MO, Robert OB, Blase AC, John PE, et al. (2014) AHA/ACC guideline for the management of patients with valvular heart disease: A report of the American College of Cardiology/American Heart Association Task Force on Practice Guidelines. Circulation 129.

2 Cohen HA, O'Neill BP (2015) TMVR: Continuing the paradigm shift in valvular heart disease: Hype or hope? J AM Coll Cardiol 66: 1020-1022.

3 Kiss D, Anwaruddin S (2017) Recent clinical trials in valvular heart disease. Curr Opin Cariol 32: 343-347.

4 Cheung A, Webb J, Verheye S, Moss R, Boone R, et al. (2014) Shortterm results of transapical transcatheter mitral valve implantation for mitral regurgitation. J Am Coll Cardiol 64: 1814-1819.

5 Condado JF (2015) Valve-in-valve transcatheter aortic and mitral replacement. Cardiac Interventions Today.

6 Seiffert M, Conradi L, Baldus S, Schirmer J, Knap M, et al. (2012) advances have emerged for alternative, less invasive treatment of mitral valve disease $[1,3]$. These novel therapies include the MitraClip for degenerative and functional mitral regurgitation (COAPT and EVEREST II trials) with successful 5-year results, and more recently TMVR therapy [1,3]. TMVR has emerged as an offlabel alternative to surgical valve repair or replacement, with early studies reporting excellent immediate and one-year results following successful TMVR [3-7]. Recently, Muller et al. examined the use of a novel transcatheter mitral valve in a multicentre study, and observed successful 30-day results in $93 \%$ of patients with absence of mortality and stroke [8]. In the past couple of years, simultaneous TAVR and TMVR has been performed, with several case reports and small studies demonstrating excellent short-term and intermediate results $[9,10]$

\section{Conclusion}

This case demonstrates that simultaneous TAVR and off-label VIV TMVR is a reasonable alternative for high-risk surgical patients with stenotic native and failing bioprosthetic valves. Future research should examine long-term outcomes of simultaneous TAVR and TMVR compared to alternative interventions. The increased implantation of surgical bioprosthetic valves and increased risk of repeat surgical valve replacements for the aging population will inevitably lead to further use of this procedure.

Transcatheter mitral valve-in-valve implantation in patients with degenerated bioprostheses. JACC Cardiovascular Interv 5: 341-349.

7 Hasan R, Mahadevan VS, Schneider H, Clarke B (2013) First in human transapical implantation of an Inverted Transcatheter Aortic Valve Prosthesis to Treat Native Mitral Valve Stenosis. Circulation 128: e74-e76

8 Muller D, Farivar R, Jansz P, Richard B, Darren W, et al. (2017) Transcatheter mitral valve replacement for patients with symptomatic mitral regurgitation. J Am Coll Cardiol 69: 381-391.

9 Elkharbotly A, Delago A, El-Hajjar M (2016) Simultaneous transapical transcatheter aortic valve replacement and transcatheter mitral valve replacement for native valvular stenosis. Catheter Cardiovasc Interv 87: 1347-1351.

10 Witkowski A, Kuśmierski K, Chmielak Z, Dąbrowski M, Jastrzębski J, et al. (2015) First-in-man simultaneous transcatheter aortic and mitral valve replacement to treat severe native aortic and mitral valve stenoses. JACC Cardiovasc Interv 8: 1399-1401. 